\title{
miR-185-3p regulates the invasion and metastasis of nasopharyngeal carcinoma by targeting WNT2B in vitro
}

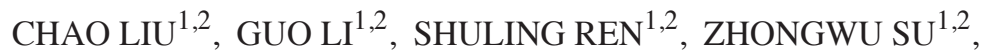 \\ YUNYUN WANG ${ }^{1,2}$, YONGQUAN TIAN ${ }^{1,2}$, YONG LIU $^{1,2}$ and YUANZHENG QIU ${ }^{1,2}$ \\ ${ }^{1}$ Department of Otolaryngology Head and Neck Surgery; ${ }^{2}$ Otolaryngology Major Disease Research Key \\ Laboratory of Hunan, Xiangya Hospital Central South University, Changsha, Hunan 410008, P.R. China
}

Received August 27, 2015; Accepted January 6, 2017

DOI: $10.3892 / \mathrm{ol} .2017 .5778$

\begin{abstract}
MicroRNAs (miRs) have been recognised as important regulators of malignant behaviour in different types of human cancer, including nasopharyngeal carcinoma (NPC). A previous study by our group revealed that miR-185-3p regulates the radioresistance of NPC cells. The present study aimed to investigate the effect of miR-185-3p on NPC invasion and metastasis. Human NPC CNE-2 and 5-8F cell lines were transfected with a miR-185-3p mimic and miR-185-3p inhibitor, respectively, and their effects on the invasion and metastasis of these cells was assessed using a wound healing assay and Matrigel invasion assay. The target gene of miR-185-3p, Wnt family member 2B (WNT2B) was silenced in $5-8 \mathrm{~F}$ cells using siRNA in order to investigate its function in NPC. Data from the present study demonstrated that the expression of miR-185-3p was the highest in 5-8F and lowest in CNE-2 cells out of a range of NPC cell lines. Following the transfection of miR-185-3p mimic into CNE-2 cells, the wound healing and Matrigel invasion assays indicated that the migration and invasion ability of CNE-2 cells was significantly reduced compared with the negative control group. In addition, the inhibition of miR-185-3p in 5-8F cells significantly increased the capacity for migration and invasion. Furthermore, silencing WNT2B expression resulted in a significant reduction in the invasion and metastasis in $5-8 \mathrm{~F}$ cells. The inhibition of miR-185-3p, which promotes invasion and metastasis, could be reversed through the silencing of WNT2B in 5-8F cells. The results of the present study indicate that miR-185-3p mediates the invasion and metastasis of NPC by targeting WNT $2 B$ in vitro.
\end{abstract}

Correspondence to: Dr Yong Liu or Professor Yuanzheng Qiu, Department of Otolaryngology Head and Neck Surgery, Xiangya Hospital Central South University, 87 Xiangya Road, Changsha, Hunan 410008, P.R. China

E-mail: liuyongent@csu.edu.cn

E-mail:xyqyz@hotmail.com

Key words: nasopharyngeal carcinoma, miR-185-3p, Wnt family member 2B, metastasis

\section{Introduction}

Nasopharyngeal carcinoma (NPC) is a type of malignant epithelial carcinoma of the head and neck that frequently occurs in Southern China and South-Eastern Asia (1). Despite advances in radiotherapy, chemotherapy and biological therapy, the overall 5-year survival rate for NPC remains poor $(2,3)$. The ability of NPC to metastasize contributes to the poor treatment efficacy (4). Therefore, investigations into the molecular mechanism underlying the metastasis of NPC are required, to aid in the development of novel therapeutic strategies and improve the prognosis of patients with NPC.

microRNAs (miRNAs/miRs) are small non-coding RNA molecules of between 19 and 25 nucleotides in length (5). They can interact with the untranslated or coding regions of mRNAs via base paring, which results in alterations in protein expression (6,7). Evidence indicates that human miRNAs can function as oncogenes or tumour suppressors, depending on their target genes, in various malignancies (8). In NPC, multiple miRNAs have been reported to be associated with multiple malignant behaviours, including increased cell proliferation (9), evasion of apoptosis (10), radioresistance (11) and chemoresistance (12). Regarding cell invasion and tumour metastasis, miRNAs, such as miR-744 and miR-26a, can enhance or suppress these activities in NPC by targeting Rho GTPase activating protein 5 or enhancer of zeste 2 polycomb repressive complex 2 subunit, respectively $(13,14)$. These findings suggest that miRNAs provide a novel area for the study of NPC invasion and metastasis.

miR-185-3p is defined as the mature miRNA of the precursor miR-185, which is generated from the $3^{\prime}$ side (15). In a previous study by our group, it was revealed that miR-185-3p could regulate the radiosensitivity of NPC cells by targeting the gene Wnt family member 2B (WNT2B) (15). However, the role of miR-185-3p in the invasion and metastasis of NPC remained unclear. Therefore, the present study aimed to investigate the association between miR-185-3p and the invasion and metastasis of NPC.

\section{Materials and methods}

Cell lines and culture conditions. Human NPC cell lines (CNE-1, CNE-2, HNE-1, HNE-2 and 5-8F) were purchased 
from the Cell Centre of Central South University (Changsha, China). The cells were cultured in RPMI-1640 medium (Hyclone; GE Healthcare Life Sciences Logan, UT, USA) supplemented with $10 \%$ foetal bovine serum (FBS) (Gibco; Thermo Fisher Scientific, Inc., Waltham, MA, USA) at $37^{\circ} \mathrm{C}$ in a humidified atmosphere with $5 \% \mathrm{CO}_{2}$. Cells in the exponential stage of growth were used for subsequent experiments.

Transfection of miR-185-3p mimic or inhibitor. CNE-2 and $5-8 \mathrm{~F}$ cells were seeded (both at a density of $2 \times 10^{5}$ cells/well) into 6-well plates and transfected with an miR-185-3p mimic or inhibitor and negative control (all chemically synthesized by Shanghai GenePharma Co., Ltd., Shanghai, China) using Lipofectamine ${ }^{\circledR} 2000$ Transfection reagent (Invitrogen; Thermo Fisher Scientific, Inc.) according to the manufacturer's protocol. The sequences for miR-185-3p mimic, inhibitor and negative control were as follows: mimic, sense, 5'-AGGGGC UGGCUUUCCUCUGGUC-3' and antisense, 5'-CCAGAG GAAAGCCAGCCCCUUU-3'; inhibitor, 5'-GACCAGAGG AAAGCCAGCCCCU-3'; mimic negative control, sense, 5'-UUCUCCGAACGUGUCACGUTT-3' and antisense, 5'-ACGUGACACGUUCGGAGAATT-3'; inhibitor negative control, 5'-CAGUACUUUUGUGUAGUACAA-3'. The transfection efficiency was assessed using fluorescence microscopy via carboxyl fluorescein labelled miRNA and miRNA expression was evaluated using reverse transcription-quantitative polymerase chain reaction (RT-qPCR) analysis.

$R T$-qPCR analysis. To quantify miRNA expression, total miRNA was extracted using the miRNeasy Mini kit (Qiagen, Inc., Valencia, CA, USA). The All-in-One ${ }^{\mathrm{TM}}$ miRNA RT-qPCR Detection kit (GeneCopoeia, Inc., Rockville, MD, USA) was used to reverse transcribe miRNAs and detect the expression of mature miRNAs according to the manufacturer's protocol. The amount of miRNA used for reverse transcription was $~ 100 \mathrm{ng}$ and this was diluted 5 times for the PCR reaction. Primers for miR-185-3p were purchased from GeneCopoeia, Inc. (cat. no. HmiRQP0246). qPCR was conducted using the iQ5 ${ }^{\mathrm{TM}}$ Real-time PCR Detection system (Bio-Rad Laboratories, Inc., Hercules, CA, USA). miRNA PCR quantification was conducted using the $2^{-\Delta \Delta \mathrm{Cq}}$ method (16) and normalised to U6. All data are presented as the mean \pm standard deviation from experiments performed in triplicate.

Cell migration and invasion assays. Cell migration activity was evaluated using a wound healing assay. A total of $2 \times 10^{5}$ cells were seeded into 6-well plates and cultured for $48 \mathrm{~h}$ until $90 \%$ confluency, and the cell monolayer was scraped using a $10 \mu \mathrm{l}$ plastic pipette tip. The lengths of the initial gap at $0 \mathrm{~h}$ and the residual gaps at 24-72 $\mathrm{h}$ following scraping were calculated using images captured at these time points.

A Matrigel invasion assay was performed according to the manufacturer's protocol. Briefly, $2 \times 10^{4}$ cells with serum-free media were seeded into the top chamber of a two-layer plate, with a Matrigelä-coated membrane (BD Biosciences, San Jose, CA, USA) between the layers and medium containing $10 \%$ FBS was added to the lower chamber. Following a $48 \mathrm{~h}$ incubation period, non-invading cells on the upper surface of the membrane were removed. Cells on the lower surface of the membrane were fixed with $4 \%$ paraformaldehyde, stained with $1 \%$ crystal violet and subsequently counted in 5 randomly selected fields under an inverted microscope (Leica Microsystems GmbH, Wetzlar, Germany) at magnification x200. All experiments were performed in triplicate.

Western blot analysis. A total of $72 \mathrm{~h}$ following transfection of the cells with WNT2B siRNA (cat. no. stQ0008281, Guangzhou, RiboBio Co., Ltd., Guangzhou, China) and negative control using Lipofectamine ${ }^{\circledR} 2000$ Transfection reagent, total protein was extracted using a radioimmunoprecipitation assay lysis buffer (Beyotime Institute of Biotechnology, Shanghai, China) and equal amounts of protein $(30 \mu \mathrm{g})$ were separated using SDS-PAGE on a $12 \%$ gel and transferred onto a nitrocellulose membrane. Membranes were blocked with 5\% non-fat milk at room temperature for $1 \mathrm{~h}$. Protein expression was detected through the incubation of the membranes with a polyclonal anti-WNT2B primary antibody (dilution, 1:800; cat. no. bs-1946R; Boiss Inc., Woburn, MA, USA) at $4^{\circ} \mathrm{C}$ overnight and washed 3 times for $10 \mathrm{~min}$ each in PBS plus $0.1 \%$ Tween 20 , followed by incubation with a secondary antibody goat anti-rabbit Immunoglobulin G-horseradish peroxidase (dilution, 1:2,000; cat. no. sc-2054; Santa Cruz Biotechnology Inc., Dallas, TX, USA) for $1 \mathrm{~h}$ at room temperature. The blots were detected using enhanced chemiluminescence reagent (Beyotime Institute of Biotechnology, Shanghai, China) and exposed in a ChemiDoc MP imaging system (Bio-Rad Laboratories, Inc., Hercules, CA, USA). Anti- $\beta$-actin (dilution, 1:1,000; cat. no. sc-130656; Santa Cruz Biotechnology Inc., Dallas, TX, USA) was used as the loading control, and was also incubated $4^{\circ} \mathrm{C}$ overnight for the primary antibody and for $1 \mathrm{~h}$ at room temperature for the secondary antibody.

Statistical analysis. Data are presented as the mean \pm standard deviation. Statistical analyses were performed using a two-tailed Student's $t$-test between two samples, and a one-way analysis of variance between more than two groups, followed by Tukey's post hoc test. Analyses were performed using SPSS software (version 18.0; SPSS Inc., Chicago, IL, USA). $\mathrm{P}<0.05$ was considered to indicate a statistically significant difference.

\section{Results}

Expression of miR-185-3p in NPC cells. RT-qPCR was performed to measure the expression of miR-185-3p in NPC CNE-1, CNE-2, HNE-1, HNE-2 and 5-8F cells (Fig. 1). The results revealed that $\mathrm{CNE}-2$ cells had the lowest miR-185-3p expression and that $5-8 \mathrm{~F}$ cells had the highest miR-185-3p expression relative to U6. Therefore, these two cell lines were selected to use in subsequent experiments.

Overexpression of miR-185-3p inhibits the invasion and metastasis of CNE-2 NPC cells. An miR-185-3p mimic and negative control (vector) were transfected into CNE-2 cells. A transfection efficiency of $92.2 \pm 4.7 \%$ was achieved following observation under a fluorescence microscope (Fig. 1A). The expression of miR-185-3p (mimic) was significantly upregulated by $142.8 \pm 24.2$ times compared with the negative control group $(\mathrm{P}<0.01$; Fig. $2 \mathrm{~A})$. The wound healing assay revealed that cell migration was significantly inhibited by miR-185-3p mimic transfection compared with the negative control group 


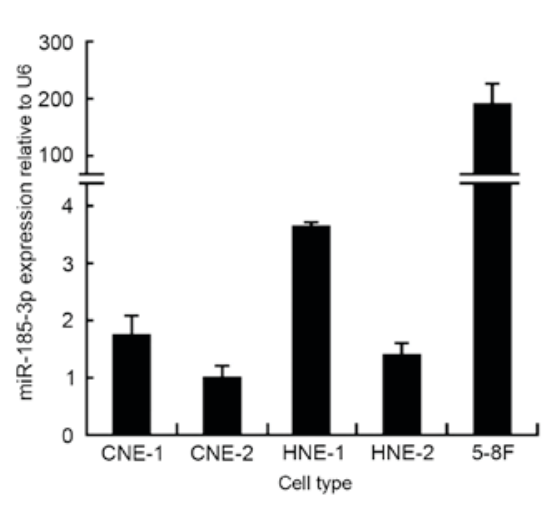

Figure 1. Expression of miR-185-3p in CNE-1, CNE-2, HNE-1, HNE-2 and $5-8 \mathrm{~F}$ nasopharyngeal carcinoma cells relative to U6. The expression of miR-185-3p was lowest in CNE-2 cells and highest in 5-8F cells. miR, microRNA.

\section{A}

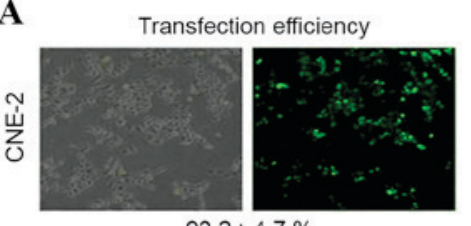

$92.2 \pm 4.7 \%$

B
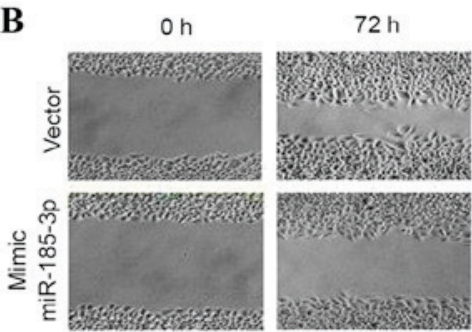

CNE-2

\section{C}
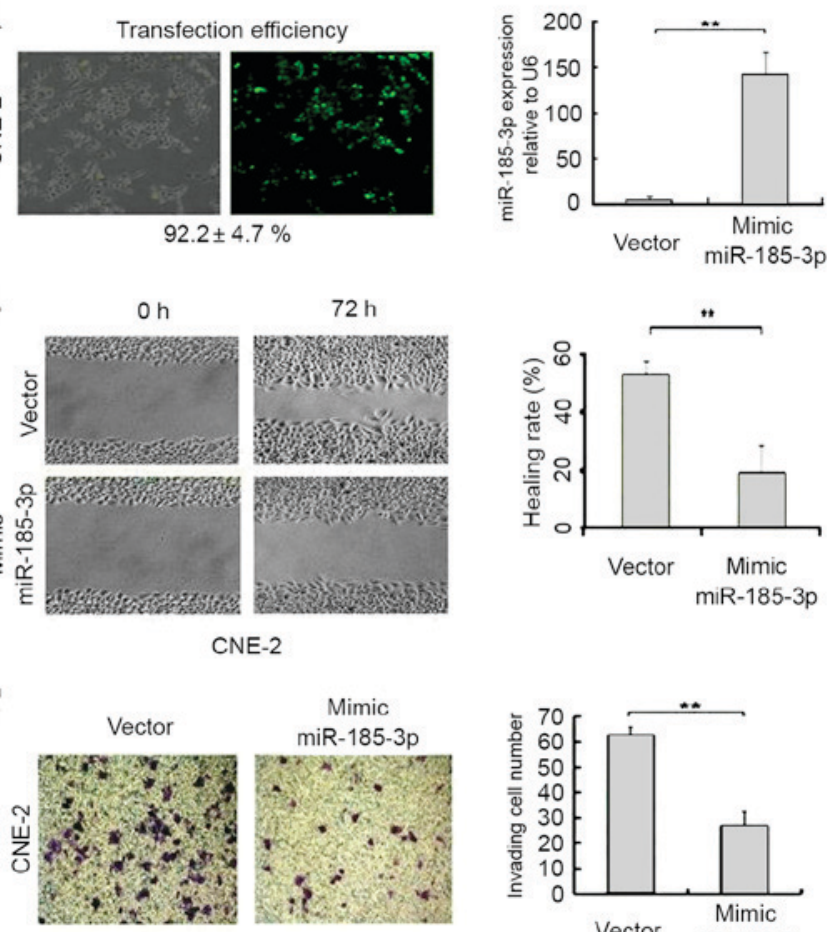

A

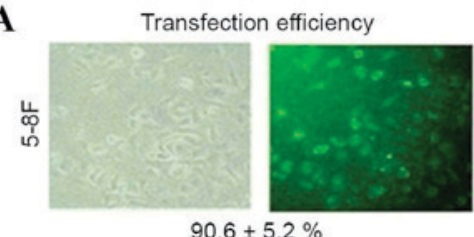

$90.6 \pm 5.2 \%$

B

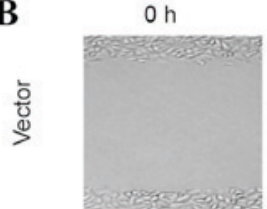

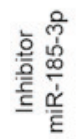

C

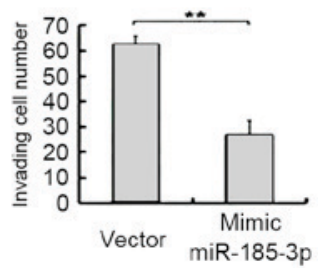
${ }^{* *} \mathrm{P}<0.01$. miR, microRNA.

Figure 2. Overexpression of miR-185-3p inhibits the invasion and metastasis of CNE- 2 cells. (A) The transfection efficiency and expression of miR-185-3p mimic were determined using a fluorescence microscope and reverse transcription-quantitative polymerase chain reaction, respectively. (B) The migration ability of cells was examined using a wound healing assay. (C) The invasion capacity of cells was investigated using a Matrigel invasion assay. Results are presented as the mean \pm standard deviation $(n=3) .{ }^{* *} \mathrm{P}<0.01$. miR, microRNA.

(healing rate, $20.7 \pm 5.8$ vs. $51.0 \pm 4.4 \%$; $\mathrm{P}<0.01$; Fig. $2 \mathrm{~B}$ ). The Matrigel invasion assay demonstrated that the number of invading cells was significantly decreased in miR-185-3p mimic-transfected cells compared with the negative control group (29.7 \pm 7.1 vs. $62.9 \pm 5.7$ invading cells; $\mathrm{P}<0.01$; Fig. $2 \mathrm{C})$. These results demonstrate that the overexpression of miR-185-3p significantly inhibits the migration and invasion of NPC cells, suggesting that miR-185-3p expression has an antimetastasis effect.
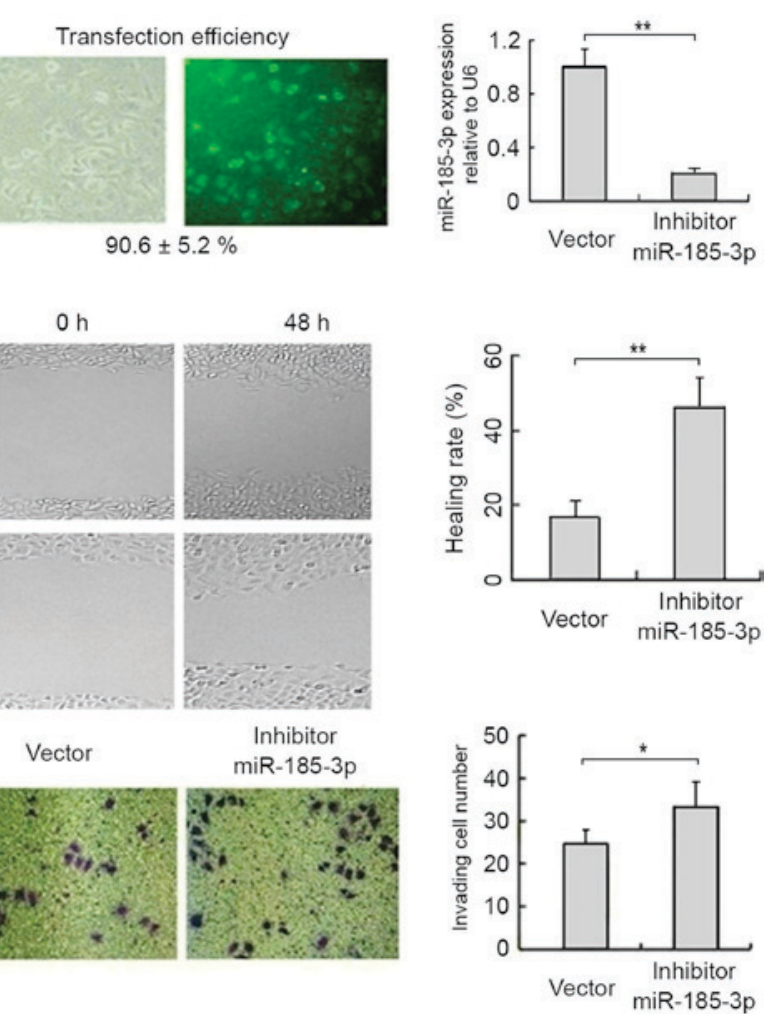

Figure 3. Inhibition of miR-185-3p increases the invasion and metastasis of 5-8F cells. (A) The transfection efficiency of miR-185-3p inhibitor and expression of miR-185-3p were determined using a fluorescence microscope and reverse transcription-quantitative polymerase chain reaction, respectively. (B) The migration of cells was examined using a wound healing assay. (C) The invasion of cells was examined using a Matrigel invasion assay. Results are presented as the mean \pm standard deviation $(n=3) .{ }^{*} \mathrm{P}<0.05$,

Inhibition of miR-185-3p increases the invasion and metastasis of 5-8F NPC cells. The transfection efficiency of the miR-185-3p inhibitor was $90.6 \pm 5.2 \%$ and miR-185-3p expression was significantly inhibited by $78.4 \pm 3.6 \%$ compared with the negative control group (both $\mathrm{P}<0.01$; Fig. $3 \mathrm{~A}$ ). The wound healing assay demonstrated that cell migration was significantly increased in cells that had been transfected with the miR-185-3p inhibitor compared with the negative control group (healing rate, $46.6 \pm 7.8$ vs. $17.0 \pm 4.4 \%$; $\mathrm{P}<0.01$; Fig. $3 \mathrm{~B}$ ). The Matrigel invasion assay demonstrated that the number of invading cells was significantly increased in the miR-185-3p inhibitor-transfected cells compared with the negative control group (32.2 \pm 6.7 vs. $21.0 \pm 3.5$ invading cells; $\mathrm{P}<0.05$; Fig. $3 \mathrm{C}$ ). These results indicate that miR-185-3p expression increases the invasion and metastasis of NPC cells.

WNT2B affects the invasion and metastasis of NPC cells. miRNAs exert their function through their effect on target genes. In a previous study by our group, $W N T 2 B$ was validated as a target gene of miR-185-3p (15). Thus, the role of $W N T 2 B$ expression on the invasion and metastasis of NPC was examined by silencing its expression in NPC cells. A WNT2B siRNA and negative control (vector) were transfected into 5-8F cells. Western blot analysis demonstrated that the Wnt2B protein was downregulated in the WNT2B siRNA-transfected cells compared with the control group (Fig. 4A). In addition, the migration ability of 5-8F 

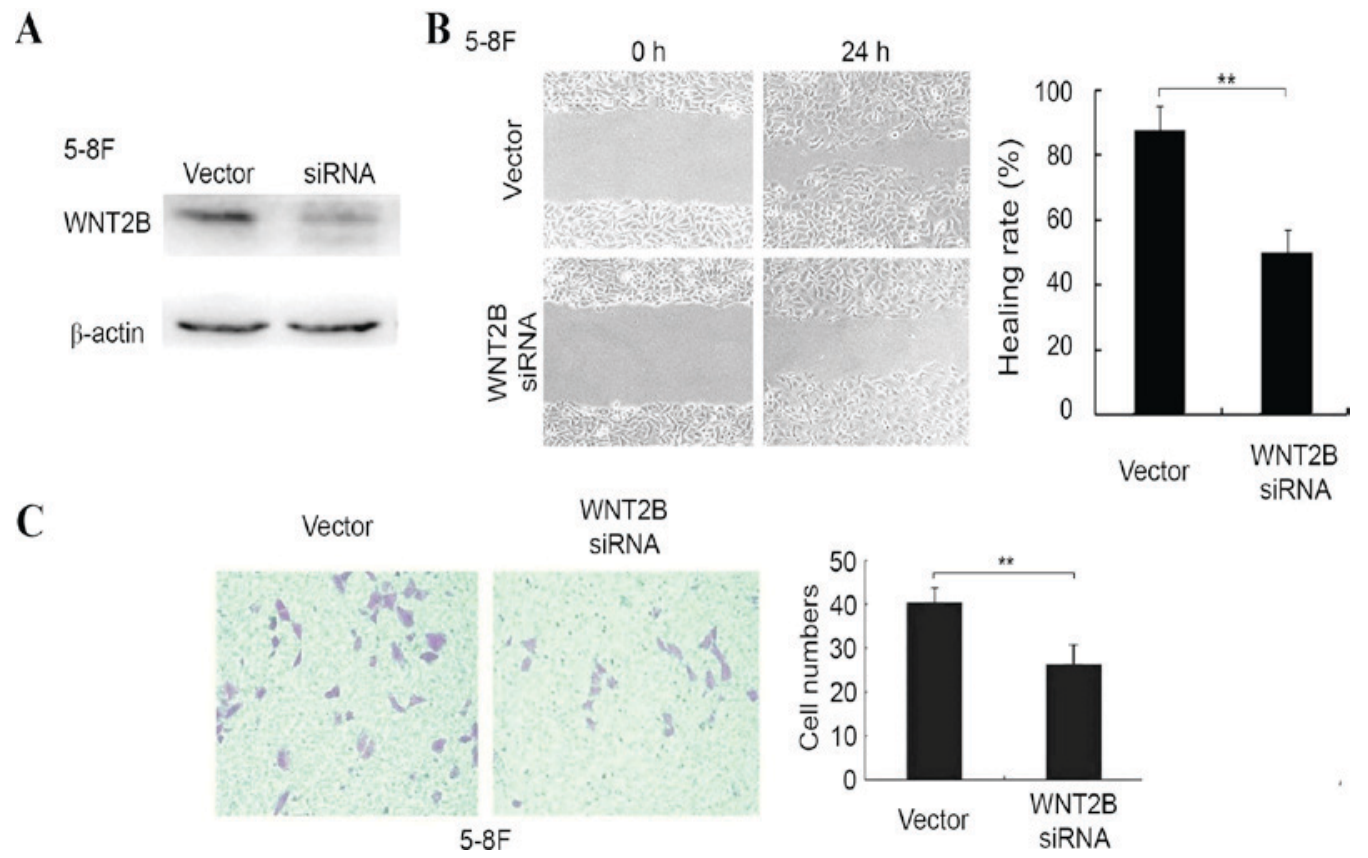

Figure 4. WNT2B silencing affects the invasion and metastasis of 5-8F nasopharyngeal carcinoma cells. (A) WNT2B was silenced by siRNA. (B) The migration of cells was examined using a wound healing assay. (C) The invasion capacity of the cells was examined using a Matrigel invasion assay. Results are presented as the mean \pm standard deviation $(\mathrm{n}=3) .{ }^{* *} \mathrm{P}<0.01$. siRNA, small interfering RNA; WNT2B, Wnt family member $2 \mathrm{~B}$.

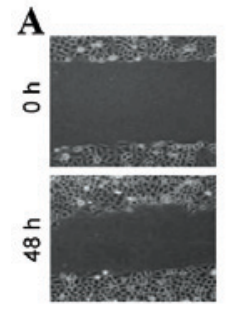

B

Vector

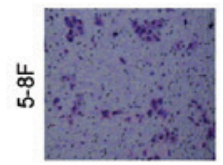

Vector
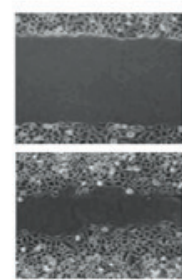

Inhibitor miR-185-3p

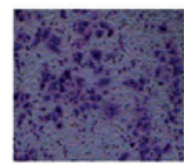

Inhibitor miR-185-3p

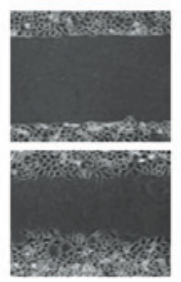

Inhibitor miR-185-3p/ WNT2B SIRNA

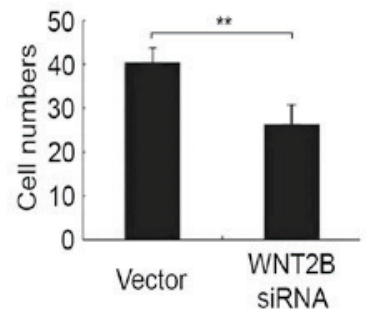

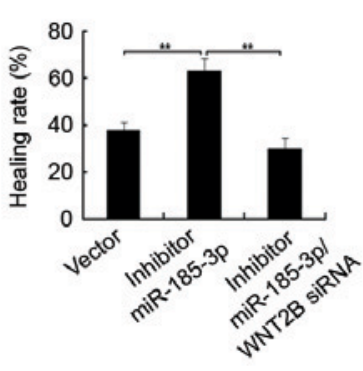

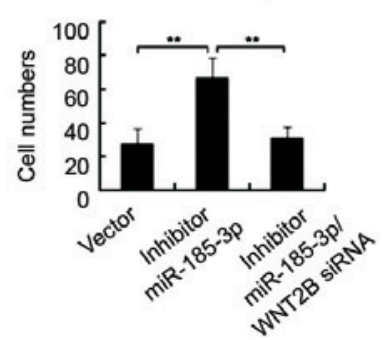

Figure 5. Downregulation of WNT2B reverses the effect of miR-185-3p inhibition on the invasion and metastasis of 5-8F NPC cells. (A) The migration of different cell groups was examined using wound healing experiments. (B) The invasion of different cell groups was examined using Matrigel invasion assays. Results are presented as the mean \pm standard deviation $(\mathrm{n}=3) .{ }^{* *} \mathrm{P}<0.01$. miR, microRNA; siRNA, small interfering RNA; WNT2B, Wnt family member $2 \mathrm{~B}$.

cells was significantly inhibited in WNT2B siRNA-transfected cells compared with the control group $(50.7 \pm 6.9$ vs. $87.9 \pm 7.4 \%$, $\mathrm{P}<0.01$; Fig. 4B). Furthermore, significantly less invading cells were observed in $W N T 2 B$-silenced cells compared with the control group (26.4 \pm 4.4 vs. $40.4 \pm 3.3$ invading cells; $\mathrm{P}<0.01$; Fig. 4C). These results demonstrate that silencing of $W N T 2 B$, a target gene of miR-185-3p, significantly inhibits the invasion and metastasis of NPC cells.

Downregulation of WNT2B reverses the effect of $\mathrm{miR}-185-3 p$ inhibition on the invasion and metastasis of 5-8F NPC cells. To further investigate the effects of miR-185-3p and $W N T 2 B$ on the invasion and metastasis of NPC, WNT2B-targeting siRNA was transfected into 5-8F cells that had also been transfected with an miR-185-3p inhibitor. Cells were also transfected with the vector or miR-185-3p inhibitor alone. The wound healing assay revealed that the significantly increased ability for cell migration following transfection with the miR-185-3p inhibitor ( $\mathrm{P}<0.01$ vs. the vector control group) was significantly reversed through $W N T 2 B$ silencing (healing rate, $63.0 \pm 5.5 \%$ vs. $29.7 \pm 5.1 \%$; $\mathrm{P}<0.01$ ) (Fig. 5A). The Matrigel invasion assay demonstrated that the significantly increased number of invading cells following 
miR-185-3p inhibitor transfection $(\mathrm{P}<0.01 \mathrm{vs}$. the vector control group) could be significantly reversed by silencing $W N T 2 B$ $(62.9 \pm 5.5$ vs. $29.6 \pm 5.1 ; \mathrm{P}<0.01)$ (Fig. $5 \mathrm{~B})$. These results demonstrate that the downregulation of $\mathrm{miR}-185-3 \mathrm{p}$, which promotes the invasion and metastasis of NPC cells, could be partially reversed through the silencing of $W N T 2 B$.

\section{Discussion}

Understanding the molecular mechanisms underlying NPC invasion and metastasis is essential for the development of improved therapeutic strategies to treat patients with NPC. With the development of functional genomics, tumour regulatory factors, such as miRNAs, have gradually attracted more attention. miRNAs have been reported to serve an important role in various malignant behaviours, including the invasion and metastasis of NPC (17). A previous study by our group on NPC radioresistance-associated miRNA profiles revealed that miR-185-3p was downregulated in NPC radioresistant cells. Additionally, it was confirmed that miR-185-3p could regulate the radioresistance of NPC by targeting WNT2B in vitro $(15,18)$. The results of the present study indicate that miR-185-3p can target $W N T 2 B$ to affect the invasion and metastasis of NPC cells. This has aided in the elucidation of the complex molecular mechanisms underlying the invasion and metastasis of NPC.

An miRNA precursor molecule is used to generate two mature miRNAs from the $3^{\prime}$ and $5^{\prime}$ end, and for miR-185, it was named as miR-185-3p and miR-185-5p, respectively. At present, the majority of studies on miR-185 have focused on miR-185-5p, a tumour suppressor associated with various aspects of cancer progression, such as proliferation, evasion of apoptosis, cell cycle progression and chemoresistance (19-21). Furthermore, miR-185-5p can inhibit colorectal cancer cell metastasis via targeting stromal interaction molecule 1 and regulate breast cancer cell invasion by targeting vascular endothelial growth factor A $(22,23)$. Regarding the function of miR-185-3p, a previous study demonstrated that miR-185-3p is differentially expressed under hypoxic stress in soft tissue sarcoma cells and that it may be associated with endotoxin-induced myocardial injury (24). Maussion et al (25) reported that miR-185-3p targets neurotrophic tyrosine kinase receptor 2-T1, the downregulation of which is associated with the neurobiological process underlying suicide. Additionally, miR-185-3p can bind to v-myc avian myelocytomatosis viral oncogene homolog in the cellular growth signalling pathway, inhibiting cell proliferation and arresting cell cycle progression (26). Functional analyses of the role of miR-185-3p in malignant tumour behaviours were performed in our previous study that investigated NPC radioresistance (15). In the present study, the role of miR-185-3p in NPC invasion and metastasis was investigated, with results indicating that similarly to miR-185-5p, miR-185-3p functions as a tumour suppressor.

miRNAs primarily pair with the mRNAs of target genes to regulate malignant cancer behaviour. In our previous study, it was identified that $W N T 2 B$ is the target gene of miR-185-3p (15). WNT2B, an important oncogene that encodes a component of the Wnt signalling pathway, is located on human chromosome $1 \mathrm{p} 13.2$ and is upregulated in various malignant tumours $(27,28)$. In pancreatic cancer, increasing $W N T 2 B$ expression is significantly correlated with perineural invasion and predicts a poor overall survival rate of patients (29). Furthermore, WNT2B can upregulate the expression of cyclin D1 and $\beta$-catenin, and increases the chemoresistance of oral cancer cells to cisplatin (30). Additionally, silencing WNT2B can inhibit the metastasis of ovarian cancer cells through epithelial-mesenchymal transition (EMT) and the Akt serine/threonine kinase signalling pathway in vitro (31). These findings illustrate that Wnt $2 \mathrm{~B}$ serves an important role in tumour malignancy. However, to the best of our knowledge, the function of Wnt2B in NPC, regarding its association with NPC radioresistance, has only been reported by our group $(15,32)$. In the current study, it was revealed that Wnt2B increases the invasion and metastasis of NPC cells in vitro, which supports future functional studies into the role of WNT2B in NPC.

Investigating the molecular mechanisms underlying the involvement of miR-185-3p and $W N T 2 B$ in NPC invasion and metastasis aids in the understanding of the pathological progression of NPC. In our previous study, miR-185-3p and $W N T 2 B$ were demonstrated to regulate the expression of the EMT biomarkers E-cadherin and vimentin (15). EMT is considered to be a classical mechanism for tumour invasion and metastasis (33). In addition, EMT is associated with the activation of the Wnt signalling pathway in various types of cancer, such as gastric, pancreatic and colorectal (34-36). Furthermore, it was demonstrated that miR-185-3p is able to alter the expression of $\beta$-catenin and glycogen synthase kinase-3 $\beta$ (15), which are essential components of the Wnt signalling pathway. The results of the current study and previous studies lead to the hypothesis that miR-185-3p and Wnt2B regulate NPC invasion and metastasis through EMT and the Wnt signalling pathway. However, further studies are required to confirm this theory.

In conclusion, the results of the present study suggest that the miR-185-3p/WNT2B axis regulates the invasion and metastasis of NPC. Therefore, this axis may be a valuable NPC-associated biomarker and a potential therapeutic target for the treatment of patients with NPC.

\section{Acknowledgements}

The present study was supported by the National Natural Science Foundation of China (grant nos. 81372426, 81202128 and 81172558), the National Natural Science Foundation of Hunan Province (grant nos. 2015JJ3137 and 14JJ2018) and the Research Fund for the Doctoral Program of Higher Education of China (grant no. 20120162120049).

\section{References}

1. Wei WI and Sham JS: Nasopharyngeal carcinoma. Lancet 365 : 2041-2054, 2005.

2. Razak AR, Siu LL, Liu FF, Ito E, O'Sullivan B and Chan K: Nasopharyngeal carcinoma: The next challenges. Eur J Cancer 46: 1967-1978, 2010.

3. Zhang L, Chen QY, Liu H, Tang LQ and Mai HQ: Emerging treatment options for nasopharyngeal carcinoma. Drug Des Devel Ther 7: 37-52, 2013.

4. Bensouda Y, Kaikani W, Ahbeddou N, Rahhali R, Jabri M, Mrabti H, Boussen $\mathrm{H}$ and Errihani H: Treatment for metastatic nasopharyngeal carcinoma. Eur Ann Otorhinolaryngol Head Neck Dis 128: 79-85, 2011. 
5. Bartel DP: MicroRNAs: Genomics, biogenesis, mechanism and function. Cell 116: 281-297, 2004.

6. Griffiths-Jones S, Grocock RJ, van Dongen S, Bateman A and Enright AJ: miRBase: MicroRNA sequences, targets and gene nomenclature. Nucleic Acids Res 34: D140-D144, 2006.

7. Gu W, Wang X, Zhai C, Zhou T and Xie X: Biological basis of miRNA action when their targets are located in human protein coding region. PLoS One 8: e63403, 2013.

8. Di Leva G, Garofalo M and Croce CM: MicroRNAs in cancer. Annu Rev Pathol 9: 287-314, 2014.

9. Pan X,Peng G,Liu S, Sun Z,Zou Zand Wu G: MicroRNA-4649-3p inhibits cell proliferation by targeting protein tyrosine phosphatase SHP-1 in nasopharyngeal carcinoma cells. Int J Mol Med 36: 559-564, 2015

10. Zhang ZC, Li YY, Wang HY, Fu S, Wang XP, Zeng MS, Zeng YX and Shao JY: Knockdown of miR-214 promotes apoptosis and inhibits cell proliferation in nasopharyngeal carcinoma. PLoS One 9: e86149, 2014.

11. Zhang T, Sun Q, Liu T, Chen J, Du S, Ren C, Liao G and Yuan Y: MiR-451 increases radiosensitivity of nasopharyngeal carcinoma cells by targeting ras-related protein 14 (RAB14). Tumour Biol 35: 12593-12599, 2014

12. Peng X, Cao P, He D, Han S, Zhou J, Tan G, Li W, Yu F, Yu J, Li Z and Cao K: MiR-634 sensitizes nasopharyngeal carcinoma cells to paclitaxel and inhibits cell growth both in vitro and in vivo. Int J Clin Exp Pathol 7: 6784-6791, 2014.

13. Fang Y, Zhu X, Wang J, Li N, Li D, Sakib N, Sha Z and Song W: MiR-744 functions as a proto-oncogene in nasopharyngeal carcinoma progression and metastasis via transcriptional control of ARHGAP5. Oncotarget 6: 13164-13175, 2015.

14. Yu L, Lu J, Zhang B, Liu X, Wang L, Li SY, Peng XH, Xu X, Tian WD and Li XP: miR-26a inhibits invasion and metastasis of nasopharyngeal cancer by targeting EZH2. Oncol Lett 5: $1223-1228,2013$

15. Li G, Wang Y, Liu Y, Su Z, Liu C, Ren S, Deng T, Huang D, Tian Y and Qiu Y: miR-185-3p regulates nasopharyngeal carcinoma radioresistance by targeting WNT2B in vitro. Cancer Sci 105: 1560-1568, 2014

16. Livak KJ and Schmittgen TD: Analysis of relative gene expression data using real-time quantitative PCR and the 2(-Delta Delta C(T)) Method. Methods 25: 402-408, 2001.

17. Tan G, Tang $X$ and Tang F: The role of microRNAs in nasopharyngeal carcinoma. Tumour Biol 36: 69-79, 2015.

18. Li G, Qiu Y, Su Z, Ren S, Liu C, Tian Y and Liu Y: Genome-wide analyses of radioresistance-associated miRNA expression profile in nasopharyngeal carcinoma using next generation deep sequencing. PLoS One 8: e84486, 2013.

19. Tang H, Liu P, Yang L, Xie X, Ye F, Wu M, Liu X, Chen B, Zhang L and Xie X: miR-185 suppresses tumor proliferation by directly targeting E2F6 and DNMT1 and indirectly upregulating BRCA1 in triple-negative breast cancer. Mol Cancer Ther 13: 3185-3197, 2014

20. Ma X, Shen D, Li H, Zhang Y, Lv X, Huang Q, Gao Y, Li X, $\mathrm{Gu} \mathrm{L}$, Xiu S, et al: MicroRNA-185 inhibits cell proliferation and induces cell apoptosis by targeting VEGFA directly in von Hippel-Lindau-inactivated clear cell renal cell carcinoma. Urol Oncol 33: 169.e1-11, 2015.
21. Li Q, Wang JX, He YQ, Feng C, Zhang XJ, Sheng JQ and Li PF: MicroRNA-185 regulates chemotherapeutic sensitivity in gastric cancer by targeting apoptosis repressor with caspase recruitment domain. Cell Death Dis 5: e1197, 2014.

22. Zhang Z, Liu X, Feng B, Liu N, Wu Q, Han Y, Nie Y, Wu K, Shi Y and Fan D: STIM1, a direct target of microRNA-185, promotes tumor metastasis and is associated with poor prognosis in colorectal cancer. Oncogene 35: 6043, 2016.

23. Wang R, Tian S, Wang HB, Chu DP, Cao JL, Xia HF and Ma X: MiR-185 is involved in human breast carcinogenesis by targeting Vegfa. FEBS Lett 588: 4438-4447, 2014.

24. Ding T, Li Y, Tang R, Zhang X, Yun Y, Li J and Wang D: Differential expression of miRNAs in myocardial tissues of rats with lipopolysaccharide- induced endotoxemia. Nan Fang Yi Ke Da Xue Xue Bao 35: 213-217, 2015 (In Chinese)

25. Maussion G, Yang J, Yerko V, Barker P, Mechawar N, Ernst C and Turecki G: Regulation of a truncated form of tropomyosin-related kinase B (TrkB) by Hsa-miR-185* in frontal cortex of suicide completers. PLoS One 7: e39301, 2012.

26. Liao JM and Lu H: Autoregulatory suppression of c-Myc by miR-185-3p. J Biol Chem 286: 33901-33909, 2011.

27. Katoh M: WNT2B: Comparative integromics and clinical applications (Review). Int J Mol Med 16: 1103-1108, 2005.

28. Katoh M: Differential regulation of WNT2 and WNT2B expression in human cancer. Int J Mol Med 8: 657-660, 2001.

29. Jiang H, Li F, He C, Wang X, Li Q and Gao H: Expression of Gli1 and Wnt2B correlates with progression and clinical outcome of pancreatic cancer. Int J Clin Exp Pathol 7: 4531-4538, 2014

30. Li SJ, Yang XN and Qian HY: Antitumor effects of WNT2B silencing in GLUT1 overexpressing cisplatin resistant head and neck squamous cell carcinoma. Am J Cancer Res 5: 300-308, 2014.

31. Wang H, Fan L, Xia X, Rao Y, Ma Q, Yang J, Lu Y, Wang C, Ma D and Huang X: Silencing Wnt2B by siRNA interference inhibits metastasis and enhances chemotherapy sensitivity in ovarian cancer. Int J Gynecol Cancer 22: 755-761, 2012.

32. Li G, Liu Y, Su Z, Ren S, Zhu G, Tian Y and Qiu Y: MicroRNA-324-3p regulates nasopharyngeal carcinoma radioresistance by directly targeting WNT2B. Eur J Cancer 49: 2596-2607, 2013

33. Ombrato L and Malanchi I: The EMT universe: Space between cancer cell dissemination and metastasis initiation. Crit Rev Oncog 19: 349-361, 2014

34. Huang J, Xiao D, Li G, Ma J, Chen P, Yuan W, Hou F, Ge J, Zhong M, Tang Y, et al: EphA2 promotes epithelial-mesenchymal transition through the $\mathrm{Wnt} / \mathrm{\beta}$-catenin pathway in gastric cancer cells. Oncogene 33: 2737-2747, 2014.

35. Xu W, Wang Z, Zhang W, Qian K, Li H, Kong D, Li Y and Tang Y: Mutated K-ras activates CDK8 to stimulate the epithelial-to-mesenchymal transition in pancreatic cancer in part via the Wnt/3-catenin signaling pathway. Cancer Lett 356: 613-627, 2015.

36. Hu TH, Yao Y, Yu S, Han LL, Wang WJ, Guo H, Tian T, Ruan ZP, Kang XM, Wang J, et al: SDF-1/CXCR4 promotes epithelial-mesenchymal transition and progression of colorectal cancer by activation of the $\mathrm{Wnt} / \beta$-catenin signaling pathway. Cancer Lett 354: 417-426, 2014. 